\title{
Towards standard benchmarks and reference data for validation and improvement of numerical simulation in sheet metal forming
}

\author{
Abel D. Santos ${ }^{\mathrm{a}, *}$, J. Ferreira Duarte ${ }^{\mathrm{a}}$, Ana Reis $^{\mathrm{a}}$, A. Barata da Rocha ${ }^{\mathrm{a}}$, \\ Luis Menezes ${ }^{\mathrm{b}}$, Marta Oliveira ${ }^{\mathrm{b}}$, Alain $\mathrm{Col}^{\mathrm{c}}$, T. Ono ${ }^{\mathrm{d}}$ \\ ${ }^{a}$ FEUP-DEMEGI-Department of Mechanical Engineering and Industrial Management, Porto University, \\ R. Roberto Frias, 4200-465 Oporto, Portugal \\ ${ }^{\mathrm{b}}$ FCTUC-Department of Mechanical Engineering, University of Coimbra, Pólo II Pinhal de Marrocos, 3030 Coimbra, Portugal \\ ${ }^{\mathrm{c}}$ SOLLAC-Av.Tilleuls, F-57191 Florange, France \\ ${ }^{\mathrm{d}}$ FAMOTIK, Ltd., 3F Kyouhan kudan Bldg., 1-5-10 Iidabashi, Chiyoda-ku, Tokyo 102-0072, Japan
}

Received 16 December 2001; accepted 24 January 2002

\begin{abstract}
The last decade has witnessed many advances and a lot of improvement in FE codes for simulation of sheet metal forming processes. Such advances could be followed mainly by benchmarks proposed in Numisheet conferences. It was possible to notice that the scatter of results among numerical codes has decreased so significantly that recently scattering of experimental results among different corporations was evident. However in order to pursue further developments and validate numerical results it is fundamental to have reliable reference experimental data. This is one of the objectives of a current IMS project called 3DS-Digital Die Design System.

In this paper such objectives are presented as well as some of the proposed benchmarks. It is intended to show part of the developed work concerning tool design and manufacturing methodology. Also an experimental case study about the use of piercing holes in parts and the use of counter-punch is presented. Finally some simulation results are also shown concerning one of the proposed benchmarks.

(C) 2002 Elsevier Science B.V. All rights reserved.
\end{abstract}

Keywords: Benchmark; Experimental data; Sheet metal forming; Numerical simulation; FEM; 3DS

\section{Introduction}

The present work is being developed under a project called 3DS-Digital Die Design System [1]. One of the main goals of this project is to improve the ability of finite element simulation codes to predict forming defects, especially those related to the final geometry of sheet metal parts. Nowadays breakage and defects like wrinkles are predicted by numerical simulation; however other defects like camber, warping and springback are not so easily and accurately predicted.

Since numerical development needs validation, this project includes a strong experimental line of work which will/ is being carried out in order to have reference experimental data for such purpose. Eight geometries have been selected,

\footnotetext{
Corresponding author.

E-mail addresses: abel@fe.up.pt (A.D. Santos),

luis.menezes@mail.dem.uc.pt (L. Menezes),

alain.col@sollac.usinor.com (A. Col),

ono@ns.famotik.co.jp (T. Ono).
}

in which a particular kind of defect is prone to be obtained. Each of these geometries will be used as a benchmark for comparison between numerical and experimental results.

Four of these geometries may be obtained/stamped by using the same tool basis and exchanging the active parts (punch, die, blank holder (BH) and counter-pad). This tool is the one described in this paper. Concerning geometries only two have been used herein; in the experimental work, a geometry which is prone to $2 \mathrm{D}$ springback has been used, Figs. 1-4, and in the numerical work, a geometry prone to 3D springback has been used, Fig. 16.

Due to the usual spread on experimental results in previous benchmarks [2], special care is devoted to material characterisation and standardisation of experimental procedures.

In this paper, besides the design of the tool to be used in the experimental work, an experimental study of the influence of positioning holes in parts, needed as reference points for its profile measurement, and also a numerical study related with using an additional component in tool, a counter-pad, which is needed when punching holes parts are presented. 


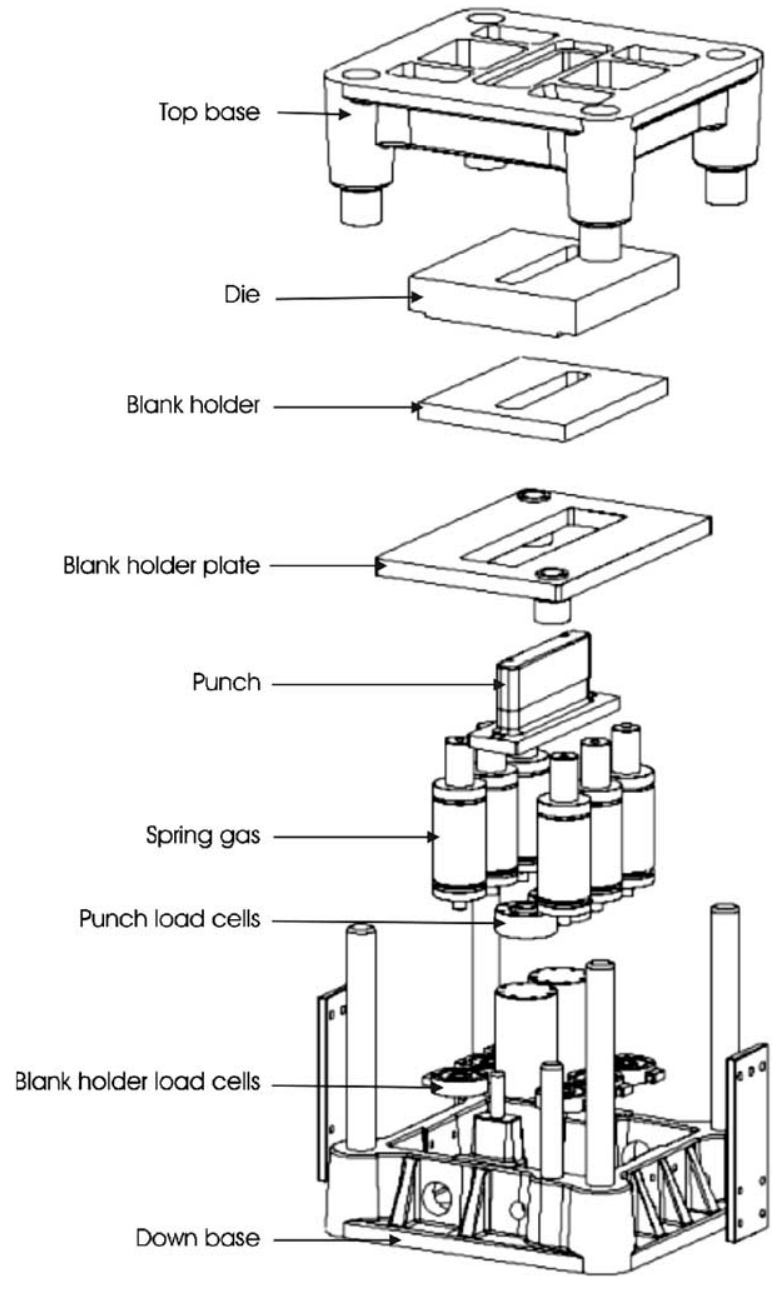

Fig. 1. Exploded view of experimental tool.

\section{Experimental tools}

In order to obtain a representative set of reference experimental results, which will be used to validate numerical results, it has been defined that different working groups would perform several identical tests with distinct but identical tools in different institutions. A special tool was

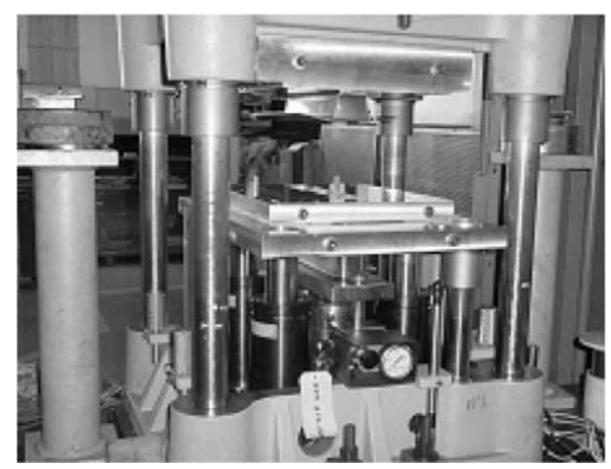

Fig. 2. Experimental tool.

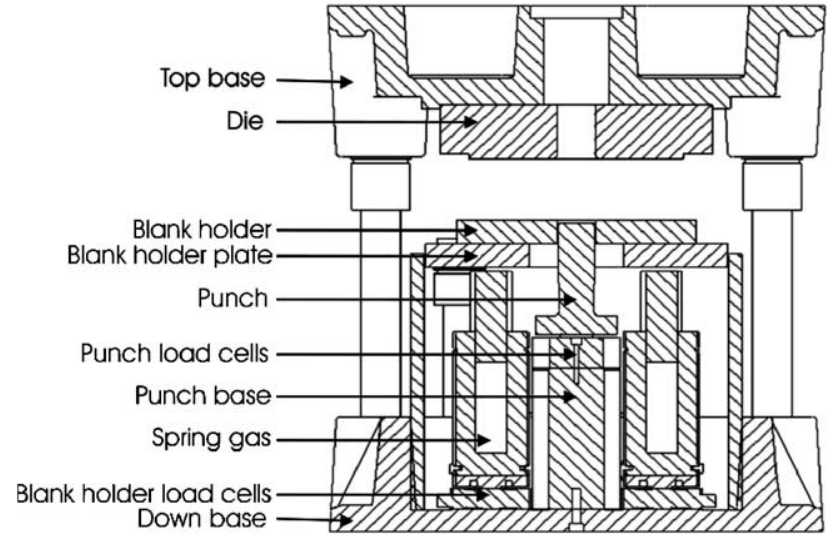

Fig. 3. Description of tool.

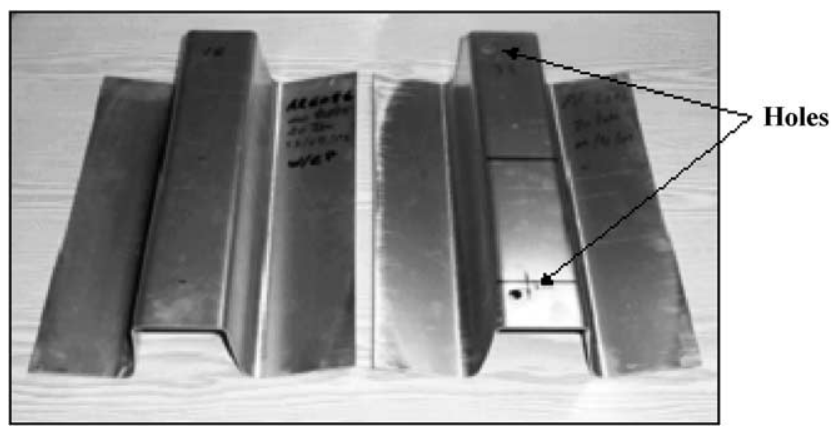

Fig. 4. Part without holes and part with holes.

designed and delivered to these different institutions. The tool main characteristics are:

- a common basis, which may receive four sets of active parts (punch, die, $\mathrm{BH}$ and counter-pad) corresponding to four different stamping geometries;

- BH force obtained from six gas springs connected among them, in order to try to obtain the smallest possible $\mathrm{BH}$ force variation during stamping;

- load cells under each gas spring and under the punch, as well as a displacement transducer so that it is possible to obtain punch force and $\mathrm{BH}$ force versus punch displacement;

- possibility of piercing two holes in stamped parts, which may be needed as reference points for part measurement.

Such a tool is described in Figs. 1 and 3 and it is shown in Fig. 2.

\section{Preliminary experimental study-influence of piercing parts}

The main objective of the experimental work is to provide reference data, which will be mainly obtained from measurement of stamped parts. Therefore, it is very important to have or define a procedure in which measurements performed by different institutions may be compared. One 


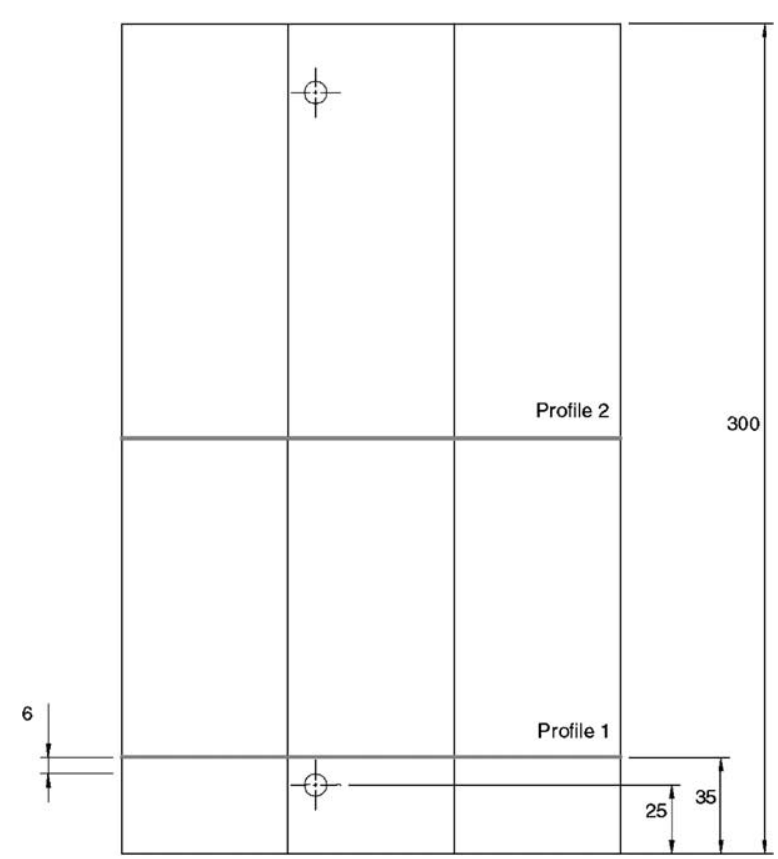

Fig. 5. Profiles to be measured (top view).

possibility is to use a positioning system ("gabarit" or jig), Figs. 5 and 6, where stamped parts will be placed and consequently will have the possibility of a well defined reference position. This procedure needs the stamped parts to have two holes, for accurate placement on the positioning system.

Since this piercing is an extra operation, beyond the intended stamping process and it is not to be numerically modelled, this presented study intends to understand the "damage" of such piercing in the final geometry of stamped parts.

In Fig. 4 is shown the selected geometry for this study. Two profiles have been measured as shown in Fig. 5, one defined $6 \mathrm{~mm}$ from the hole and the other at the centre of the part. Such profiles were measured for parts with holes and also for parts without holes, so that the influence of such piercing may be seen.

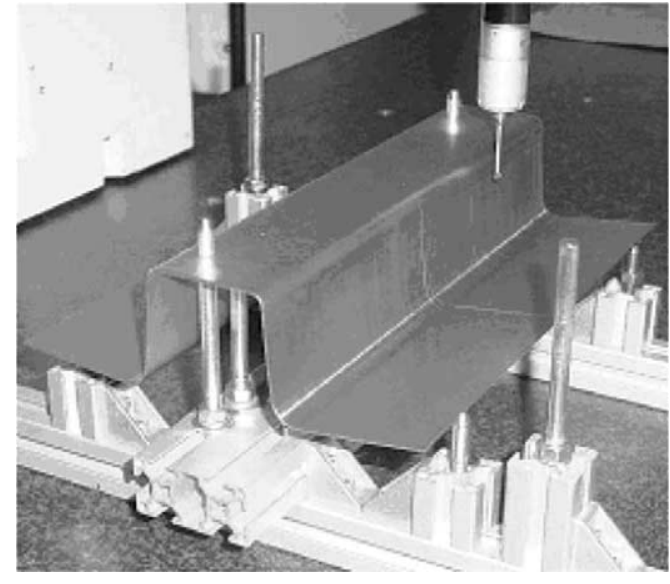

Fig. 6. Device designed for positioning parts.

\subsection{Results}

Five tests were carried out, with piercing and without piercing of the part. The selected material has been aluminium alloy, 6016. Figs. 7 and 8 show profiles 1 and 2, respectively, obtained for samples in which holes have been performed.

In Figs. 9 and 10 the same profiles are presented but for samples with no holes.

In each of these figures (Figs. 7-10) the stamping conditions have been the same. Also, in each figure the measurements for each sample have a common point $(x=70$, $y=20$ ), so that we may see the dispersion of results (the right circles in the figures). Such dispersion of results varies from 0.45 to $1.14 \mathrm{~mm}$. Fig. 11 compares profile 1 for all samples with and without holes, while Fig. 12 makes the same comparison but for profile 2 .

Values of the $x$ coordinate for all samples and the two profiles may be better understood in Fig. 13. We may see that for each profile there is an increment of $x$ value when holes are performed in the stamped part, a tendency which is

Profile1 - with Holes

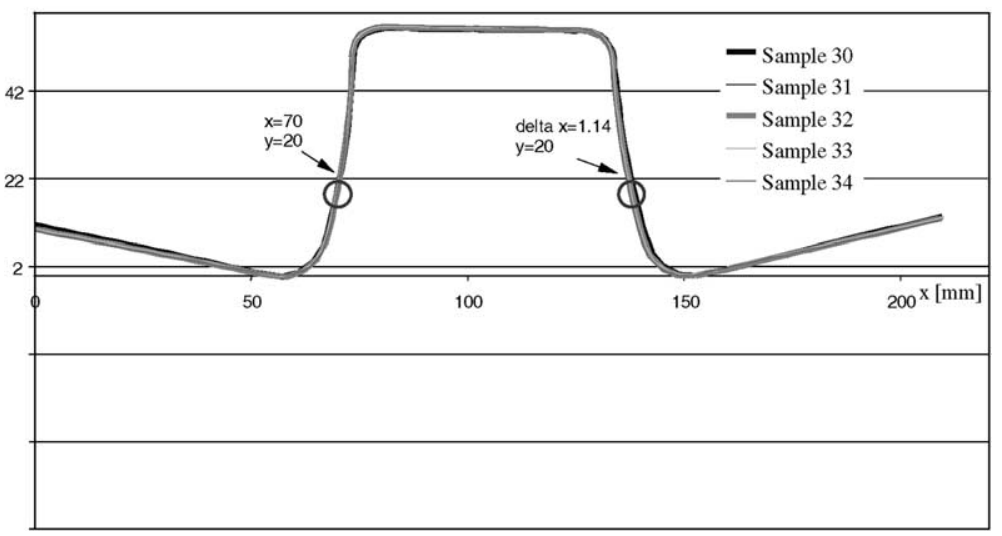

Fig. 7. Profile 1 measurements (with holes). 


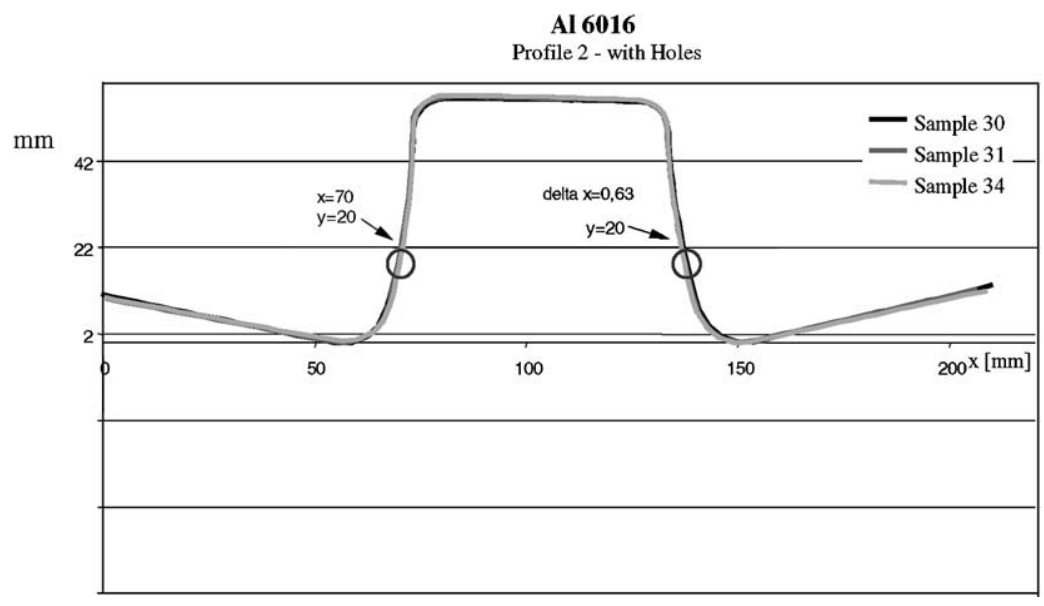

Fig. 8. Profile 2 measurements (with holes).

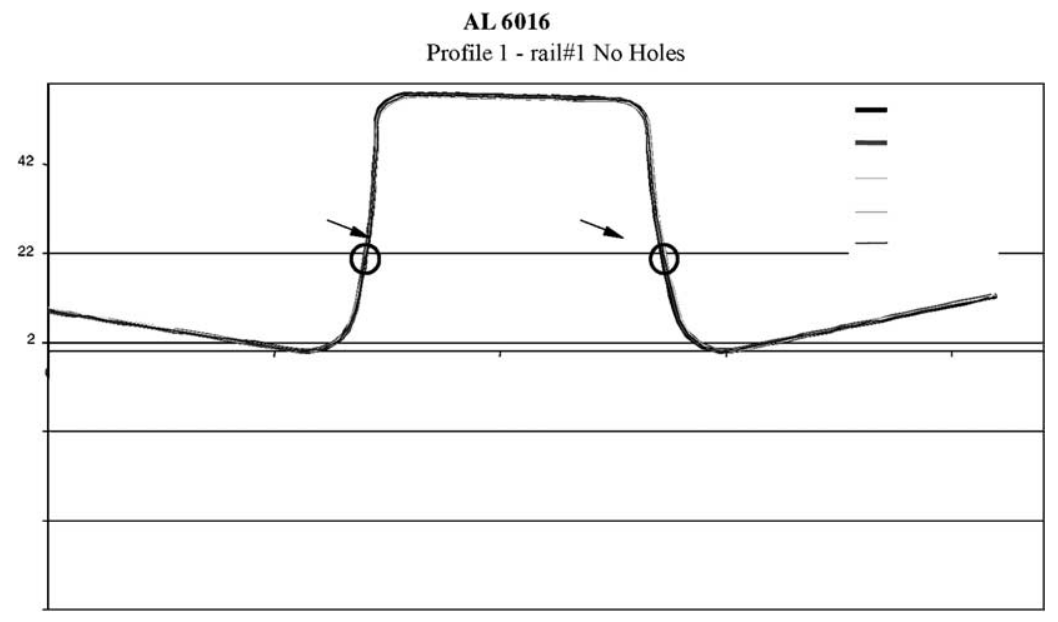

Fig. 9. Profile 1 measurements (no holes).

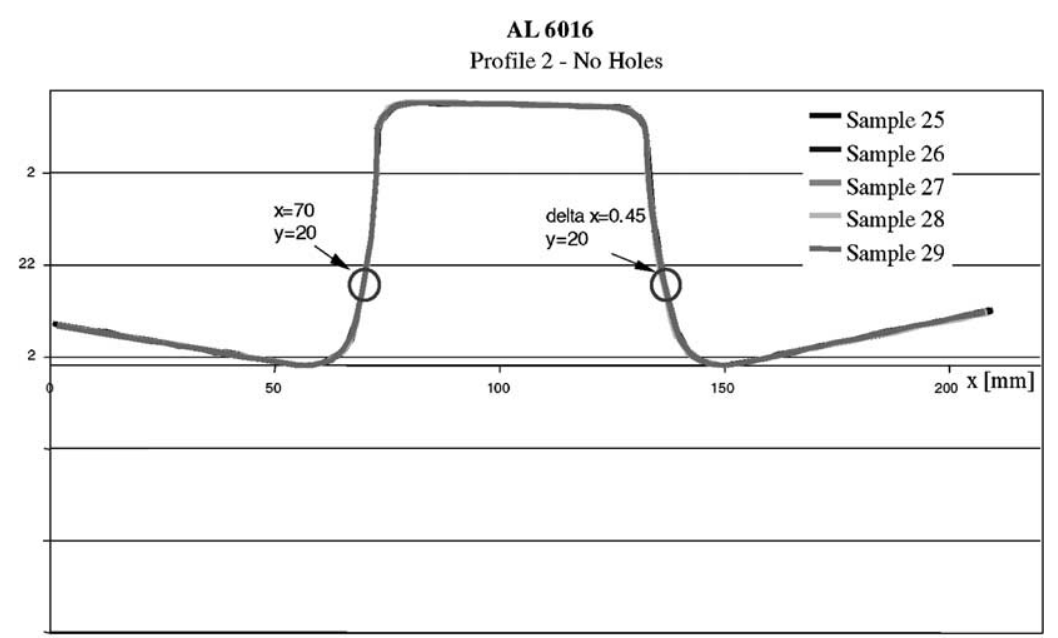

Fig. 10. Profile 2 measurements (no holes). 


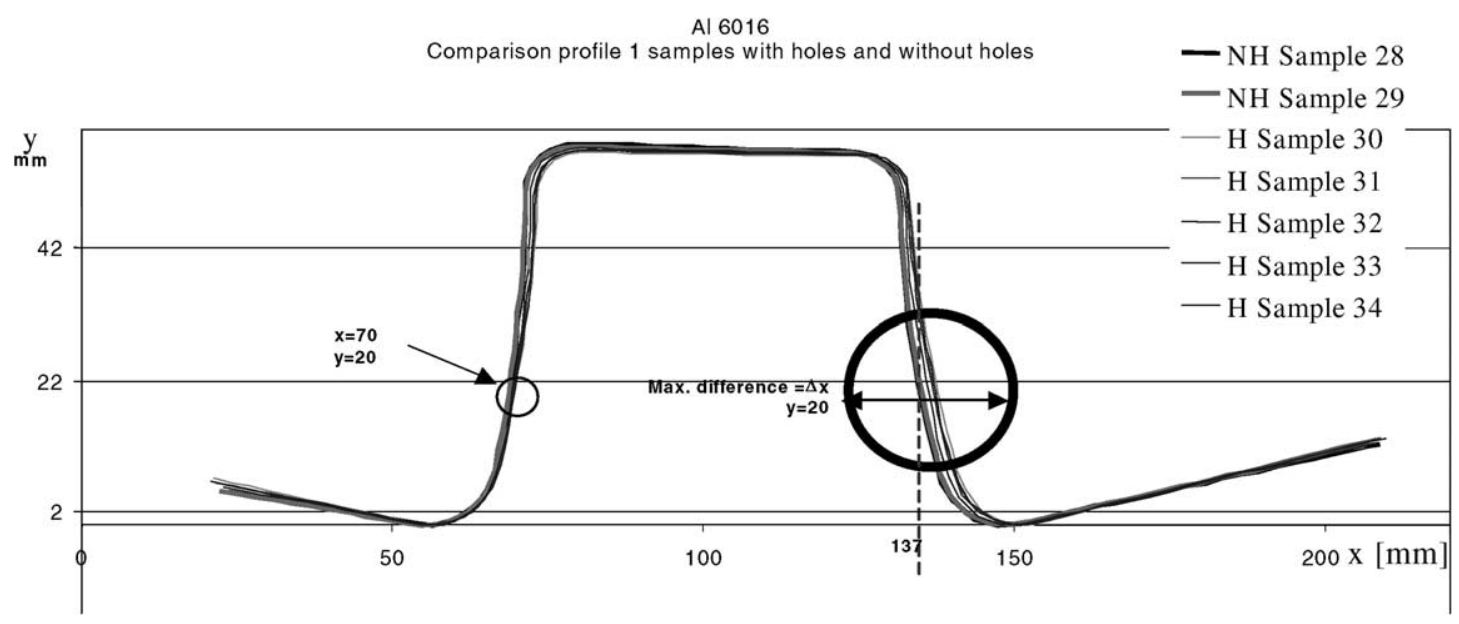

Fig. 11. Profile 1: comparison of all measurements.

Al 6016

Comparison profile 2 Samples with holes and without holes

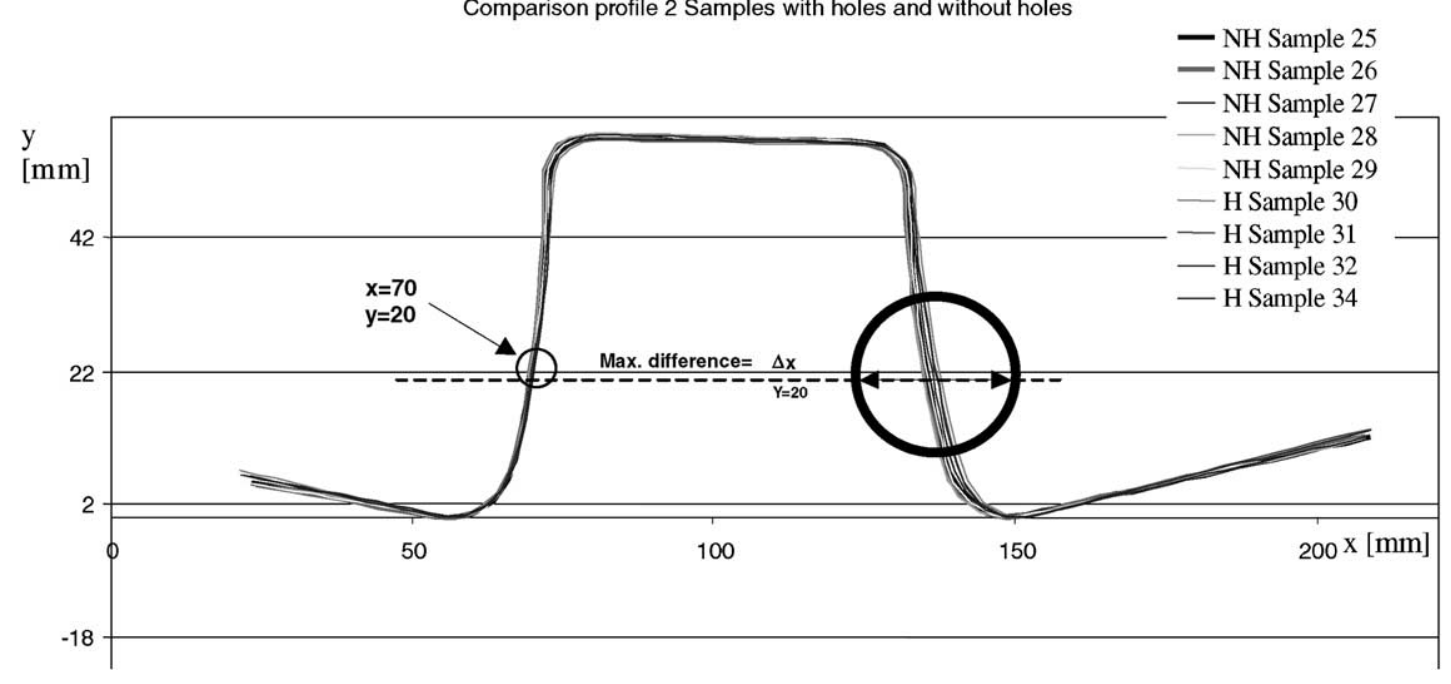

Fig. 12. Profile 2: comparison of all measurements.

similar for both profiles. If we only see profile 1 results (Fig. 14) we may see that piercing the component (making two holes) will "open" its shape by an average value of $1 \mathrm{~mm}$. However, the dispersion of results may have a similar value of magnitude $(\Delta x=1.14 \mathrm{~mm})$. Considering the total

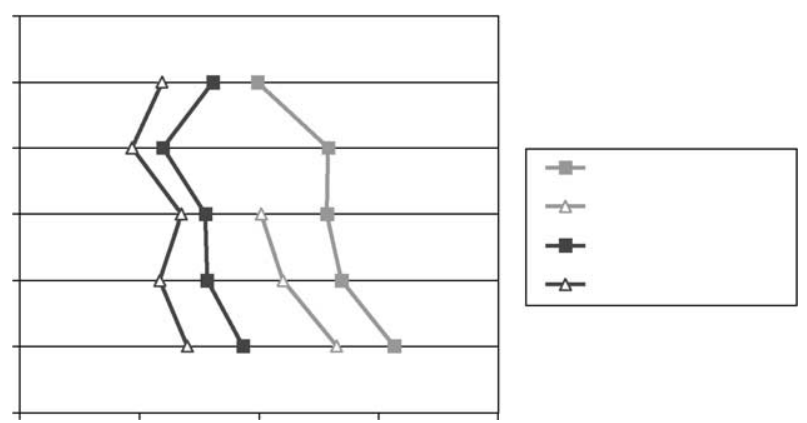

Fig. 13. $x$ coordinate for all samples at point $y=20$ (Figs. 11 and 12). width of the profile, $67 \mathrm{~mm}$, this difference in the average corresponds to a low value. Therefore, the difference in measured results due to the influence of piercing operation is very close to the spread found in the measurements.

\section{Preliminary numerical study—influence of counter-pad force}

Each geometry in the 3DS project was chosen so that it characterizes a special sheet metal forming defect. For this study the selected geometry is shown in Fig. 15, and its stamping shape is prone to develop 3D springback.

In order to perform the piercing of the part, a supplementary tool component was introduced, a counter-pad. This tool component should not have any influence on part final geometry. Its use is only to help the piercing operation at the final stage of punch travel. 


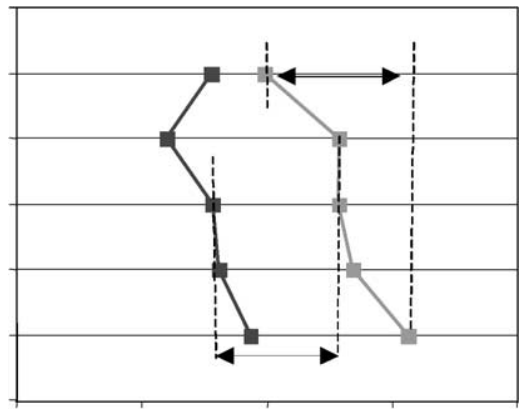

Table 1

Material properties and simulation conditions

Young's modulus

Yield strength

Swift law

Anisotropic coefficients

Width

Punch travel

Punch velocity

Friction coefficient
$21.1 \mathrm{GPa}$

$129.5 \mathrm{MPa}$

$\sigma=554(0.0016+\varepsilon)^{0.227} \mathrm{MPa}$

$r_{0^{\circ}}=2.37, r_{0^{\circ}}=2.07, r_{0^{\circ}}=3.06$

$1.0 \mathrm{~mm}$

$60 \mathrm{~mm}$

$1 \mathrm{~m} / \mathrm{s}$

0.1

Fig. 14. Comparison of results for profile 1.
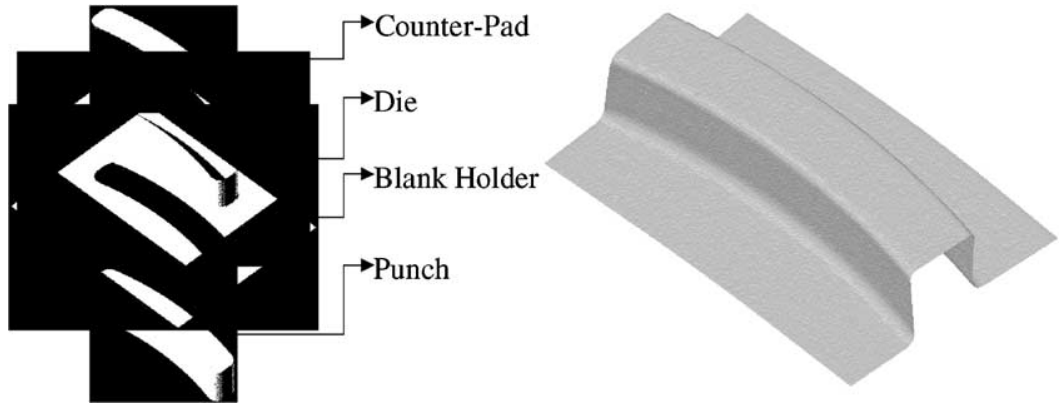

Fig. 15. Geometry of part and tool set-up.

The main goal of the present numerical study is to evaluate the influence of the piercing operation and the influence of using the counter-pad force. The results presented here refer to a steel material and its properties are presented in Table 1.
$\mathrm{BH}$ force has been defined as $20 \mathrm{t}$ and it is considered constant during stamping. This $\mathrm{BH}$ force value is the minimum to be used with this material in which the probability of defects being developed after springback is greater.

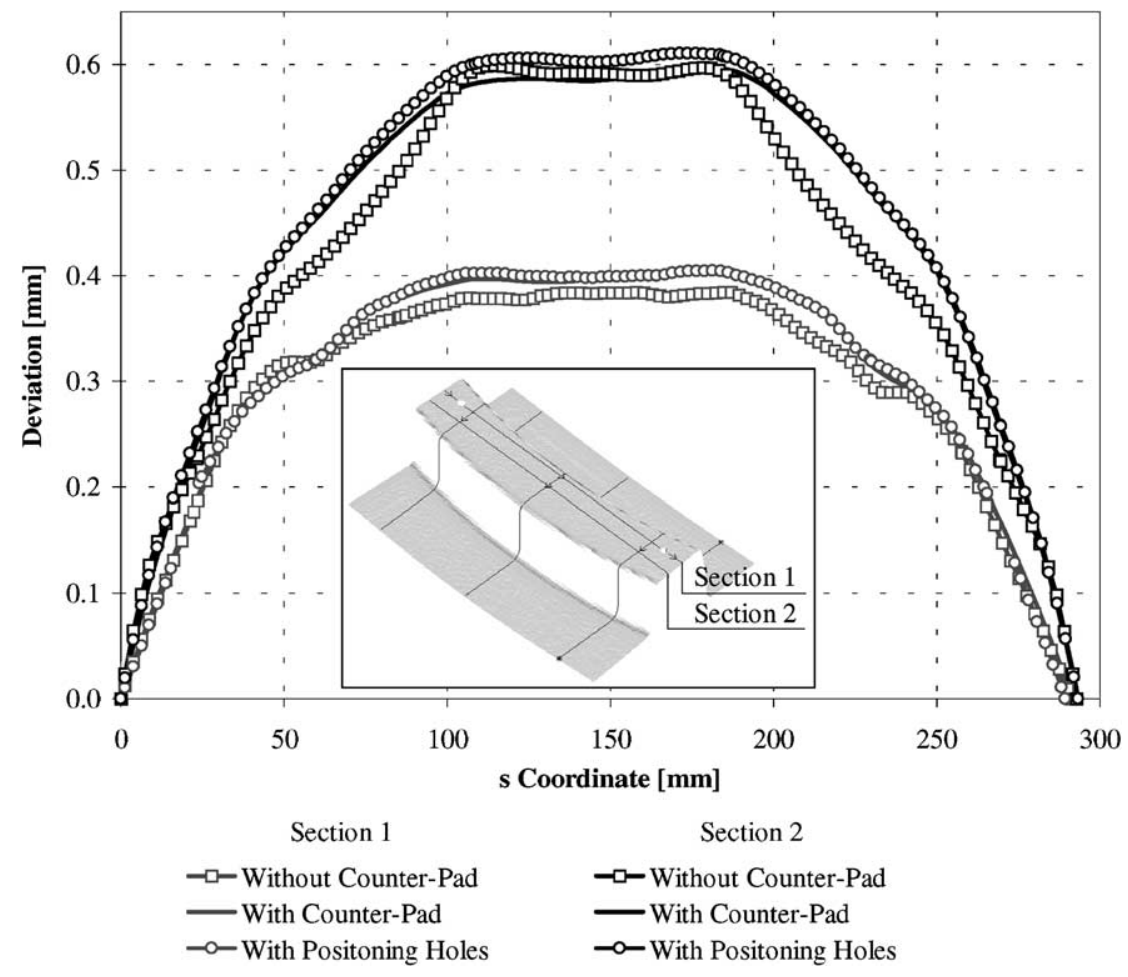

Fig. 16. $z$ coordinate in top face of part. 


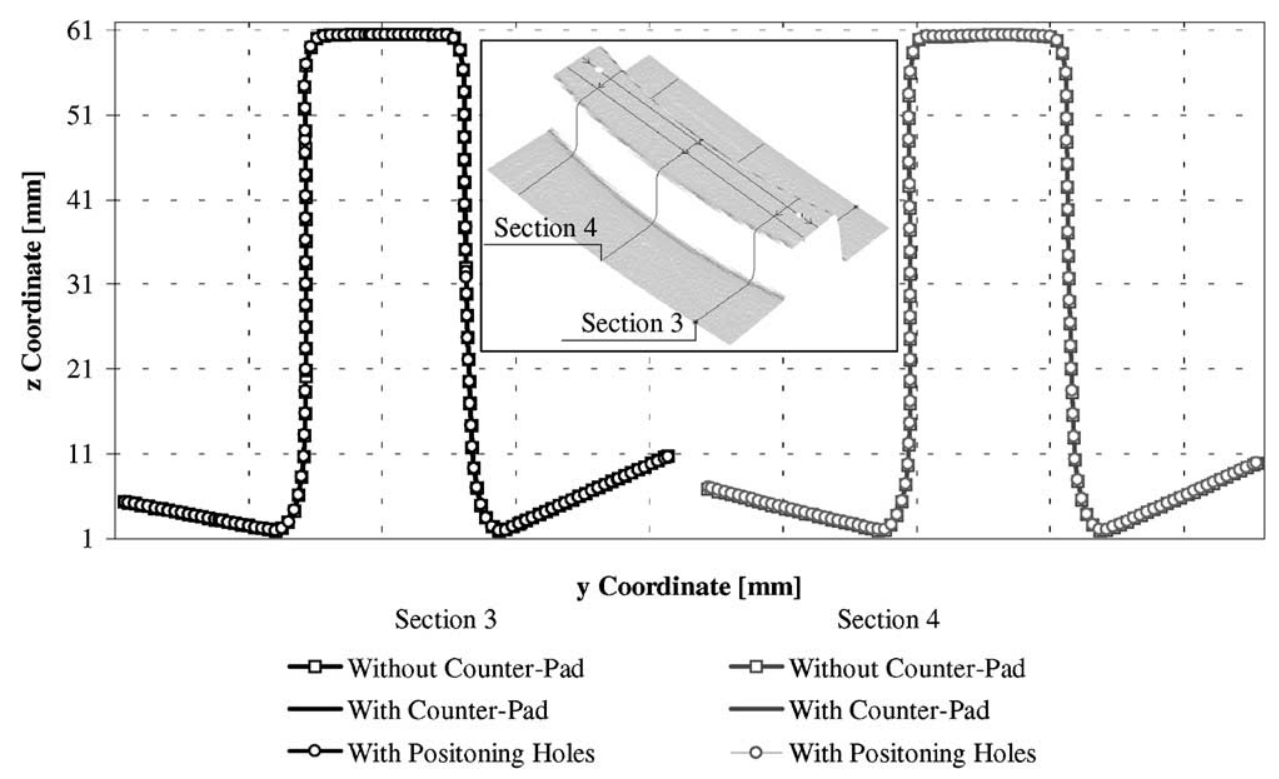

Fig. 17. Total profiles obtained: near the hole and in the middle of part.

This preliminary study was carried out using of a commercial code PAM-STAMP [3].

Three different results after springback are presented: without counter-pad, with counter-pad but without piercing and the last situation with counter-pad and piercing (before springback). Although counter-pad force is considered to be proportional to punch displacement, in simulation a constant value of $140 \mathrm{~kg}$, which corresponds to the most critical situation (maximum spring force in counter-pad) was adopted.

\subsection{Results}

Fig. 16 presents the deviation to the intended geometry on the top face of the stamped part after springback and for two different profiles. Section 1 corresponds to a longitudinal profile near the hole, while Section 2 corresponds to the central longitudinal line of the part, as shown also in Fig. 17. The larger deviation occurs in the case when the counter-pad is used since it prevents the wrinkling in the top of the component. However, the value for this deviation is very small, almost negligible, its maximum value being close to $0.04 \mathrm{~mm}$.

Fig. 17 compares the transverse profiles obtained after springback. For the profile corresponding to Section 3 the lateral borders of the sheet geometry are different, due to different flow of material. Even for central profile, Section 4, such a tendency is observed.

Fig. 18 presents the sheet thickness distribution after springback, for those three different situation defined previously. No remarkable difference was observed between the situations. Holes are located where change in thickness is not evident; therefore their existence does not seem to affect this kind of result.

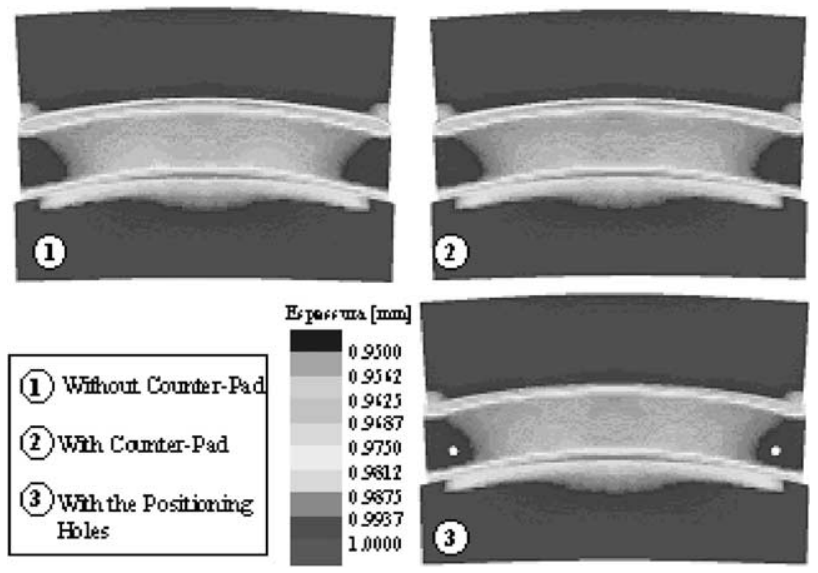

Fig. 18. Thickness distribution.

\section{Conclusions}

An experimental tool has been presented, which is intended to be used to obtain reference experimental data for validation of developments in numerical modelling of sheet metal forming processes.

An experimental study has been performed to show the influence of piercing small holes in parts, needed as reference points for measurement. It has been shown that such piercing has a small influence in final geometry of parts; such piercing operation shows a tendency to increase the angle of the side walls of the part. However, such influence is very small and its value is close to the dispersion of results obtained, when keeping all process variables and conditions unchanged.

The numerical results confirm that the piercing operation does not have an influence on the final results. Therefore, in 
numerical simulation work it is not necessary to include such piercing modelling.

\section{References}

[1] A. Col, Presentation of the 3DS research project, IDDRG 2001, Espoo, Finland, 2001
[2] J.C. Gelin, P. Picart (Eds.), Proceedings of the Fourth International Conference on Numerical Simulation of 3D Sheet Forming Processes (NUMISHEET'99), Vol. 2, University of France-Comté and ENSMM, Besançon, France, 1999.

[3] PSI, Software Company of ESI Group, Solver Reference Manual, Pam System International, S.A., 1998. 